\title{
ANÁLISE DAS ATITUDES DE ENFERMEIROS E ESTUDANTES DE ENFERMAGEM NA PARAÍBA-BR QUANTO À UTILIZAÇÃO DO COMPUTADOR
}

Sérgio Ribeiro dos Santos ${ }^{1}$

Santos SR dos. Análise das atitudes de enfermeiros e estudantes de enfermagem na Paraíba-BR quanto à utilização do computador. Rev Latino-am Enfermagem 2001 novembro-dezembro; 9(6):56-61.

O objetivo deste estudo foi analisar as atitudes dos enfermeiros e estudantes de Enfermagem em relação ao uso do computador na prática de Enfermagem. $O$ instrumento aplicado constou de dados demográficos e escala de atitudes. Para os enfermeiros, foram identificados três fatores: barreiras em relação ao computador; o computador como ferramenta de trabalho; a eficiência da informação para a Enfermagem. Para os estudantes, além destes, foi identificado mais um: necessidade de educação em informática aplicada à Enfermagem. As atitudes dos enfermeiros e estudantes são semelhantes. Conclui-se, portanto, que ambos têm atitudes moderadas em relação ao computador, porém os estudantes demonstram maior familiaridade.

DESCRITORES: enfermagem, informática clínica, comportamento

\section{ANALYSIS OF THE ATTITUDES OF NURSES AND NURSING STUDENTS CONCERNING COMPUTER USE IN PARAÍBA, BRAZIL}

In this study an attempt was made to analyze the attitudes of both nurses and Nursing students in relation to computer use. Demographic data and attitudinal scaling were used. Three factors were found for nurses: obstacles to computer use; the computer as a work tool; the effectiveness of information to Nursing. As to Nursing students, in addition to these three factors, another one was also found: the need for educational training in computer technology applied to Nursing. Nurses' and students' attitudes were similar. It was, therefore, concluded that both groups have moderate attitudes concerning computer use, although the students showed more familiarity.

KEY WORDS: nursing attitude, clinical information technology, nursing behavior

\section{ANÁLISIS DE LAS ACTITUDES DE LOS ENFERMEROS Y ESTUDIANTES DE ENFERMERÍA EN PARAIBA - BR EN RELACIÓN CON LA UTILIZACIÓN DEL COMPUTADOR}

El objetivo de este estudio fue analizar las actitudes de los enfermeros y estudiantes de enfermería en relación con el uso del computador en la práctica de la enfermería. El instrumento aplicado constó de datos demográficos y escala de actitudes. Para los enfermeros fueron identificados tres factores: barreras en relación al computador, el computador como herramienta de trabajo y la eficiencia de la información para la enfermería. Para los estudiantes, además de éstos fue identificado uno más: la necesidad de educación en informática aplicada a la enfermería. Las actitudes de los enfermeros y estudiantes son semejantes. Se concluye, por lo tanto, que ambos tienen actitudes moderadas en relación con el computador, aunque los estudiantes demostraron mayor familiaridad.

DESCRIPTORES: enfermería, informática clínica, comportamiento

\footnotetext{
${ }^{1}$ Enfermeiro, Professor Mestre em Enfermagem do Centro de Ciências da Saúde da Universidade Federal da Paraíba. Doutorando em Ciências da Saúde/ UFPB, e-mail: s.r.santos@uol.com.br.
} 


\section{INTRODUÇÃO}

Atitude pode ser definida como uma reação ou maneira como cada pessoa responde, favoravelmente ou não, a determinados objetos ou situações. Atitudes podem predizer a intenção de um indivíduo para desenvolver um comportamento em relação ao objeto conhecido ${ }^{(1)}$.

É amplamente aceito que atitudes positivas valorizam o processo de aprendizagem. As atitudes são reflexos do estado interno do homem que afetam sua escolha de ação ou comportamento em relação a pessoas, objetos ou eventos. $O$ conhecimento das atitudes é de grande importância na determinação do aprendizado das pessoas.

Assim, o comportamento do indivíduo é influenciado pelas suas atitudes. Sabe-se que o aprendizado pode sofrer influência de atitudes, embora estas também provoquem 0 aprendizado ${ }^{(2)}$. Em outras palavras, as atitudes estimulam os indivíduos na escolha de ações e determinam o estado mental de motivação para aprender. Portanto, elas são estabelecidas pela experiência das pessoas sobre algo. Essa experiência pode, em geral, ter uma resposta positiva ou negativa para uma determinada situação e influenciar a motivação pessoal para adquirir novas habilidades. Uma atitude favorável, geralmente, eleva a motivação para estudar e para reter informações em uma dada situação. De modo oposto, uma atitude negativa pode bloquear o aprendizado e a retenção de novas informações. Logo, qualquer que seja a atitude, ela pode influenciar no desenvolvimento de comportamento que exige respostas a determinadas situações ${ }^{(3)}$. Desse modo, observa-se que há forte relação entre atitudes e aprendizagem de novas habilidades.

A habilidade profissional de enfermeiros em relação ao uso do computador, na prática assistencial e gerencial, tem sido investigada por muitos pesquisadores, especialmente, nos Estados Unidos da América e na Austrália ${ }^{(4-5)}$. A partir das pesquisas de Stronge e Brodt, que desenvolveram um instrumento para medir atitudes frente ao computador ${ }^{(6)}$, outros estudos têm sido realizados ${ }^{(7-}$ 9), apresentando-se até mesmo propostas para melhorar o referido instrumento ${ }^{(5,10)}$.

Embora o instrumento de Stronge e Brodt tenha sido testado com confiança por muitos pesquisadores, poucos estudos têm constatado sua validade e a construção subjacente das medidas. Uma equipe de pesquisadores liderada por Schiwirian, usaram 0 referido instrumento para realizar um estudo comparativo entre atitudes de estudantes e enfermeiros em relação ao computador num hospital metropolitano e identificaram três componentes: a) 0 computador e o cuidado do paciente; b) o computador e a segurança pessoal; c) atitudes gerais. Os pesquisadores concluíram que os procedimentos em relação ao uso do computador pelos enfermeiros não são unidimensionais ${ }^{(3)}$. Estudo posterior foi desenvolvido por Scarpa que identificou cinco componentes: a eficiência do computador; a eficiência da enfermagem; impacto na sociedade ou nas ações de enfermagem; limitações do computador; segurança do paciente ${ }^{(4)}$

No Brasil, os estudos acerca das atitudes dos enfermeiros e estudantes de graduação em Enfermagem em relação ao computador ainda são poucos, se comparados a países como Estados Unidos, Canadá e Austrália. Sabe-se que os cursos de graduação têm implantado, nos seus currículos, a disciplina Informática na Saúde e Enfermagem. Algumas pesquisas têm procurado avaliar a resposta desse conteúdo na formação do estudante de Enfermagem. Nesse sentido, foi desenvolvido estudo com o objetivo de investigar a opinião dos alunos de Enfermagem sobre a disciplina Informática Aplicada à Saúde. Os resultados mostraram que os estudantes consideram importante a permanência da disciplina no curso de graduação em Enfermagem ${ }^{(11)}$.

Nessa perspectiva, a questão que norteia a realização deste estudo é: Quais são as atitudes dos enfermeiros e estudantes de graduação em Enfermagem com relação à utilização do computador na prática profissional?

A relevância deste estudo reside na importância de investigar as atitudes de enfermeiros e estudantes de Enfermagem quanto às suas experiências e expectativas em relação à prática do computador. Está também na necessidade de oferecer subsídios para a formação de uma base teórica e prática capaz de enfrentar os desafios do novo milênio. Daí, a necessidade de explorar os fatores que podem contribuir para suas atitudes em relação ao computador, a fim de que enfermeiros e estudantes de Enfermagem possam conduzir-se por uma visão positiva do computador nos cuidados de saúde. Nesse sentido, o estudo pretende alcançar os seguintes objetivos:

- analisar as atitudes dos enfermeiros e estudantes de Enfermagem em relação ao uso do computador na prática de Enfermagem; - comparar as atitudes dos enfermeiros em relação aos estudantes de Enfermagem, quanto ao uso do computador.

\section{MÉTODOS}

A população foi composta por enfermeiros da rede básica de saúde, de hospitais públicos e por estudantes de graduação em enfermagem, a partir do $4^{\circ}$ período do curso pelo fato deles terem uma visão da atuação da Enfermagem na prática. A amostragem ocorreu por consentimento dos respondentes, assim como das instituições. A amostra foi composta por 157 estudantes e 85 enfermeiros. A pequena participação de enfermeiros neste estudo ocorreu em virtude da dificuldade em coletar dados desses 
profissionais no local de trabalho. Essa dificuldade também ocorreu em outros estudos, conforme está demonstrado na literatura ${ }^{(3-4)}$.

O instrumento para a coleta dos dados foi composto de um questionário constituído de duas partes: informações relacionadas às características demográficas da população; atitudes em relação ao computador. $O$ questionário foi constituído de vinte enunciados dos quais oito expressam uma atitude negativa em relação ao computador e doze expressam uma atitude positiva, com escores variando de 1 a 5 pontos para os enunciados positivos e o reverso para os enunciados negativos. 0 máximo de escores do instrumento é 100, indicando uma atitude muito positiva em relação ao computador.

Para proceder-se à coleta de dados, em primeiro lugar, foram fornecidas algumas orientações aos respondentes sobre como deveriam preencher o instrumento. Os que concordaram em participar assinaram um termo de consentimento esclarecido. A seguir, em atenção aos princípios éticos dispostos na Resolução nº 196/96, do Conselho Nacional de Saúde ${ }^{(12)}$, os pesquisadores deixaram claro que a colaboração era voluntária e os participantes não precisavam identificar-se, podendo o instrumento ser devolvido em branco.

Os dados foram analisados à luz da estatística descritiva (média, desvio padrão, variância, freqüência absoluta e relativa etc.). A análise fatorial foi uma ferramenta valiosa que conduziu à identificação dos componentes principais de análise, fazendo-se uso da rotação do tipo varimax e eigenvalue $\geq 1$. Os itens com carga fatorial igual ou superior a 0,500 foram aceitos. 0 programa SPSSIPC foi usado para conduzir o processamento estatístico do estudo ${ }^{(13)}$

\section{RESULTADOS E DISCUSSÃO}

Dos 180 questionários aplicados aos estudantes, apenas $87,2 \%$ (157) foram preenchidos e devolvidos, enquanto dos 100 questionários aplicados aos enfermeiros, houve um retorno de $85 \%$ (85).
Caracterização da amostra

Tabela 1 - Dados demográficos dos estudantes e enfermeiros, João Pessoa, 1999

\begin{tabular}{llcccc}
\hline \multirow{2}{*}{ Variáveis } & & \multicolumn{2}{c}{ Estudantes } & \multicolumn{2}{c}{ Enfemeiros } \\
& & $f$ & $\%$ & $f$ & $\%$ \\
\hline \multirow{2}{*}{ Sexo } & Masculino & 9 & 5,7 & 3 & 3,5 \\
& Feminino & 148 & 94,3 & 82 & 96,5 \\
Renda & Menos de 1 salánio & 4 & 2,5 & - & - \\
Familiar & mínimo & & & & \\
& 1 a 3 salánios mínimos & 27 & 17,2 & 14 & 16,5 \\
& 4 a 6 salánios mínimos & 53 & 33,8 & 22 & 25,9 \\
& 7 a 9 salánios mínimos & 29 & 18,5 & 19 & 22,4 \\
& Mais de 10 salánios & & & & \\
& mínimos & 44 & 28 & 30 & 35,2 \\
Média de & & 21 anos & & 35 anos \\
idade & & & & & \\
\hline
\end{tabular}

A Tabela 1 confirma, o que outros estudos já revelaram, ou seja, o predomínio do sexo feminino na Enfermagem. A renda familiar entre os estudantes concentra-se na faixa de 4 a 6 salários mínimos, com $33,8 \%$, porém a média da renda familiar foi de 3,52 salários mínimos, com desvio padrão 1,15 e variância 1,32. Isso se explica porque a maioria dos estudantes vive sob a dependência dos pais, não contribuindo com recurso financeiro para custear sua manutenção e estudos. Por outro lado, entre os enfermeiros a renda familiar de maior frequêencia situou-se acima de 10 salários mínimos $(35,2 \%)$. No entanto, a média da renda familiar foi de 3,76 salários mínimos, com desvio padrão 1,11 e variância 1,230.

Outro dado importante que a Tabela 1 revela é a média de idade. Os estudantes apresentaram idade média de 21 anos, mínimo de 17 e máximo 44 anos, com desvio padrão 2,55 e variância 6,48. Já entre os enfermeiros, a idade média foi de 35 anos, mínimo de 22 e máximo 62 anos, com desvio padrão 8,27 e variância 68,405. Percebe-se que essa categoria profissional é bastante jovem. Outros estudos têm confirmado essa característica do profissional de Enfermagem ${ }^{(9,14)}$.

Tabela 2 - Perfil dos estudantes e enfermeiros em relação ao computador, João Pessoa, 1999

\begin{tabular}{|c|c|c|c|c|c|c|c|c|}
\hline \multirow{3}{*}{ Hens } & \multicolumn{4}{|c|}{ Estudantes } & \multicolumn{4}{|c|}{ Enfermeiros } \\
\hline & \multicolumn{2}{|c|}{ Sim } & \multicolumn{2}{|c|}{ Nẫo } & \multicolumn{2}{|c|}{ Sim } & \multicolumn{2}{|c|}{ Nẫo } \\
\hline & $f$ & $\%$ & $f$ & $\%$ & $f$ & $\%$ & $f$ & $\%$ \\
\hline 1. Wocê possui microcomputador em sua residência? & 46 & 29,3 & 111 & 70,7 & 33 & 38,8 & 52 & 61,2 \\
\hline 2. Wocê lê regulamente alguma publicaçẫo sobre computaçỗo? & 28 & 27,8 & 129 & 82,2 & 33 & 38,8 & 52 & 61,2 \\
\hline $\begin{array}{l}\text { 3. Você estudou computaçẫo em alguma disciplina na } \\
\text { graduaçẫo em Enfermagem? }\end{array}$ & 4 & 2,5 & 153 & 97,5 & 3 & 3,5 & 82 & 96,5 \\
\hline $\begin{array}{l}\text { 4. Wocê se sente motivado(a) a conhecer a informática e aplicá- } \\
\text { la na Enfermagem? }\end{array}$ & 130 & 82,8 & 27 & 17,2 & 80 & 94,1 & 5 & 5,9 \\
\hline
\end{tabular}


A Tabela 2 apresenta o perfil dos estudantes e enfermeiros em relação ao computador, com base nos questionamentos. Os dados revelam que $70,7 \%$ dos estudantes e $61,2 \%$ dos enfermeiros não possuem microcomputador em seus domicílios. Essa constatação não é surpreendente, considerando-se que essa categoria profissional, em sua maioria, apresenta uma baixa renda salarial, dificultando a aquisição de um equipamento de preço elevado, em termos de custo-benefício para o uso doméstico. Além disso, o desconhecimento e a falta de uma real necessidade pessoal dessa ferramenta são fatores a ser considerados.

Por outro lado, a falta de leitura na área de informática também ficou evidenciada entre os estudantes $(82,2 \%)$ e enfermeiros $(61,2 \%)$, embora se observe que os enfermeiros têm mais oportunidade de ler regularmente alguma publicação sobre computação do que os estudantes. Isso se explica pela necessidade constatada na prática profissional do enfermeiro com a instalação de computadores nos serviços de saúde, fato que, de certa forma, levaos a buscar informações sobre tecnologia em saúde.

Observa-se ainda que tanto os estudantes $(97,5 \%)$ quanto os enfermeiros $(96,5 \%)$ não tiveram oportunidade de estudar computação durante o curso de graduação em Enfermagem. É importante ressaltar que a disciplina Informática em Enfermagem foi introduzida nos currículos dos cursos de Enfermagem no início dos anos 90 , começando pelas escolas do Sul e Sudeste, até chegar ao Nordeste e, em especial, à Paraíba. Daí, a insegurança e o desconhecimento dos estudantes e enfermeiros sobre a aplicação da informática na prática de enfermagem. Porém, um resultado animador foi revelado pelos estudantes $(82,8 \%)$ e enfermeiros $(94,1 \%)$ : o fato de se sentirem motivados a conhecer a informática e aplicá-la no dia-a-dia dos cuidados de enfermagem.

Análise da escala de atitudes

A escala de atitudes foi elaborada com vinte itens que descreviam aspectos negativos e positivos do computador na prática de Enfermagem. Não houve diferença significativa no escore médio dos resultados das duas amostras. A média dos escores dos estudantes foi de 64,78 e a variância igual a 86,34, com desvio padrão de 9,29 e coeficiente de confiabilidade alpha igual a 0,7337 para 157 casos investigados. Entre os enfermeiros, a média dos escores foi de 69,12 e variância igual a 83,99, com desvio padrão de 9,16 e coeficiente de confiabilidade alpha 0,7654 para 85 casos investigados com 20 variáveis. Este resultado valida o instrumento, pois um valor para alpha maior que 0,6000 significa que houve concordância entre 0 instrumento e a população.

O teste de significância da correlação de matrizes para a análise fatorial, o teste Bartlett's de esfericidade e a medida KaiserMeyer-Olkin-KMO foram identificados nas amostras. Em relação aos estudantes, a medida de $\mathrm{KMO}$ foi igual a 0,83 , enquanto que, para os enfermeiros, foi igual a 0,75 . Quando a KMO se aproxima do valor 1 , então há suporte para o método de análise fatorial. Por outro lado, em relação aos estudantes, o teste Bartlett's foi igual a 1.376, enquanto, para os enfermeiros, foi igual a 965,91 . Significa que houve correlação entre os itens da escala para estudantes e enfermeiros.

Essa massa de informações permitiu o uso da análise dos componentes principais, através do programa SPSSIPC ${ }^{(13)}$. Para isso, aplicou-se o método de rotação varimax sobre os itens do questionário. Somente fatores com eigenvalue maiores que 1 foram aceitos. Os itens com carga fatorial (coeficientes) acima de 0,500 foram considerados para efeito de análise dos componentes principais.

O processamento dos dados permitiu identificar entre os estudantes, conforme Tabela 3, quatro fatores com percentual de variância igual a $58,66 \%$. 0 fator 1 , denominado barreiras em relação ao computador, apresentou $25,89 \%$ de variância e eigenvalues igual a 5,179; o fator 2 contribuiu com 19,43\% de variância e eigenvalues igual a 3,887 e focaliza o computador como ferramenta de trabalho; o fator 3 compreende itens relacionados com a eficiência da informação para Enfermagem, apresentando 7,19\% de variância e eigenvalues igual a 1,439; o fator 4 reflete a necessidade de educação em informática, totalizando $6,15 \%$ de variância e eigenvalues igual a 1,232. Apenas 0 item 9 do questionário foi excluído porque não apresentou carga fatorial dentro do limite mínimo estabelecido para este estudo $(0,500)$.

Tabela 3 - Identificação das variáveis pelo método de rotação varimax com normalização Kaiser, para estudantes, João Pessoa, 1999

\begin{tabular}{|c|c|}
\hline $\begin{array}{l}\text { Estuctantes } \\
\qquad r=15 T\end{array}$ & $\begin{array}{l}\text { Carge } \\
\text { Fatorial }\end{array}$ \\
\hline \multicolumn{2}{|l|}{ Fator 1 -Barreiras em relą̧̇o ao compuador } \\
\hline 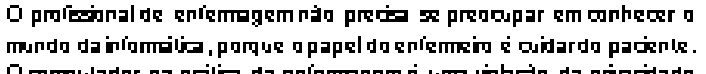 & 0,972 \\
\hline 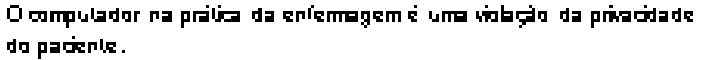 & 0,8 \\
\hline 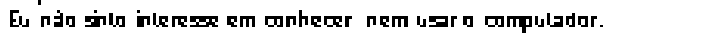 & 0.835 \\
\hline 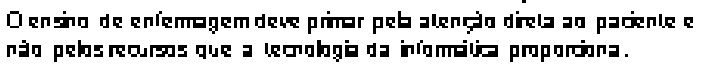 & $0, \mathbf{T}_{\mathrm{B}} \mathrm{d}$ \\
\hline Oug d & Q ו ו \\
\hline $\begin{array}{l}\text { O ommils: } \\
\text { A enisemr }\end{array}$ & $\begin{array}{l}0,750 \\
0,720\end{array}$ \\
\hline $\begin{array}{l}\text { onm qua buade. } \\
\text { A enígmagem dew }\end{array}$ & 0,870 \\
\hline \multicolumn{2}{|l|}{ 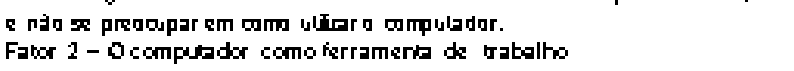 } \\
\hline 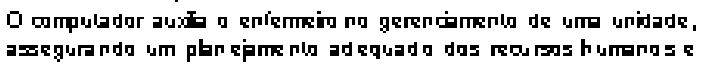 & 0,879 \\
\hline & \\
\hline 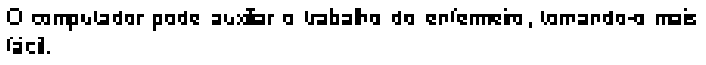 & 0,858 \\
\hline 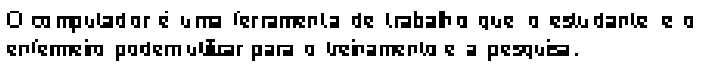 & 0,805 \\
\hline \multicolumn{2}{|l|}{ Fabor 1 - A eficiërnia d irformą̧̇o para a enkermagem } \\
\hline 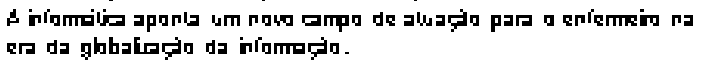 & 0,790 \\
\hline 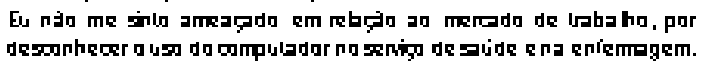 & $0,70 \theta$ \\
\hline O ommulador nàn me assola nem me embarap. & 0,895 \\
\hline 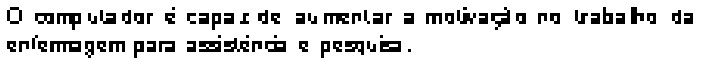 & 0.860 \\
\hline \multicolumn{2}{|l|}{$\begin{array}{l}\text { Fabor } 4 \text { - Necessidade de educa ̧ào em irfor matica aplicad a } \\
\text { erher magem }\end{array}$} \\
\hline 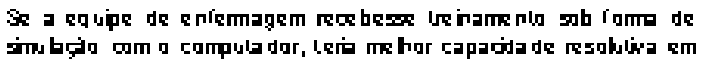 & 0,7 g \\
\hline & \\
\hline Ipulador pode mohorar a a & $0,89 d$ \\
\hline O סоmplador & (1) $\mathrm{fdd}$ \\
\hline
\end{tabular}


Em relação aos enfermeiros, conforme a Tabela 4, três fatores apresentaram percentual de variância igual a $54,28 \%$. O fator 1 também reflete as barreiras em relação ao computador, contribuindo com $30,94 \%$ de variância e eigenvalues igual a 6,189; o fator 2 aglomerou variáveis relacionadas ao computador como ferramenta de trabalho, apresentando 16,10\% de variância e eigenvalues igual a 3,222; o fator 3 compreendeu itens relacionados a eficiência da informação para a enfermagem, registrando $7,24 \%$ de variância e eigenvalues igual a 1,448. Três itens foram excluídos por apresentarem carga fatorial menor que 0,500.

Tabela 4 - Identificação das variáveis pelo método de rotação varimax com normalização Kaiser, para os enfermeiros, João Pessoa, 1999

\begin{tabular}{|c|c|}
\hline $\begin{array}{l}\text { Erfermeiros } \\
\qquad r=85\end{array}$ & $\begin{array}{l}\text { Carge } \\
\text { Fatorial }\end{array}$ \\
\hline 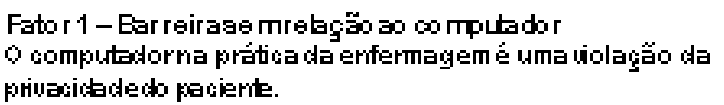 & 0,936 \\
\hline 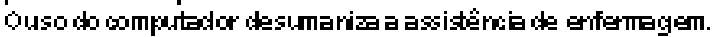 & 0,931 \\
\hline Eunẫo sinto irteresse emconhecer nem usaro computador. & 0,924 \\
\hline o computadormefazsentirconisso e incuido. & $0,85 T$ \\
\hline $\begin{array}{l}\text { Oprofissional de enfermagem nấo precisa se preocupar em } \\
\text { conhecer omundo ç informática, porcueo papeldo enfenmeino } \\
\text { é cuidardo paciente. }\end{array}$ & 0,788 \\
\hline $\begin{array}{l}\text { o ersino de enfermagem deve primarpela atenģố direta ao } \\
\text { paciente enấo pelosrecursos quea tendogia da informáica } \\
\text { proporciona. }\end{array}$ & 0,727 \\
\hline $\begin{array}{l}\text { Ánfermagem deve drecionar seus estudos naassistếncia ao } \\
\text { paciente enấo se preocupar en como urilizaro computacbr. }\end{array}$ & 0,681 \\
\hline $\begin{array}{l}\text { Ocomputador cria mais problemaspara enfermagem do que } \\
\text { aucila nodia-cha daprática profissional. }\end{array}$ & 0,681 \\
\hline $\begin{array}{l}\text { Aenfermagem nấoprecisa docomputaciorpara prestar uma } \\
\text { assistếnciacom cqualidade. } \\
\text { Fabr } 2 \text {-Oco mputador como far rame ntade trabalho }\end{array}$ & 0,606 \\
\hline $\begin{array}{l}\text { Ousodo compurtachorpode mehorara assistência prestada ao } \\
\text { paciente. }\end{array}$ & 0,798 \\
\hline 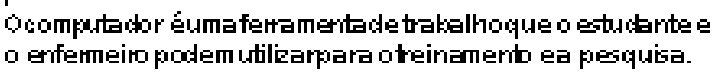 & 0,725 \\
\hline $\begin{array}{l}\text { Sea ecuipede enfermagem recebesse treirament sob forma } \\
\text { de simulaçấo com o computador, teria melhor capacidade } \\
\text { resolutiva emsituaçốs reais. }\end{array}$ & 0,715 \\
\hline $\begin{array}{l}\text { Ocomputadorpocke auxiliarotabaho doerfemeino, tomando- } \\
\text { omais fácil. }\end{array}$ & 0,621 \\
\hline 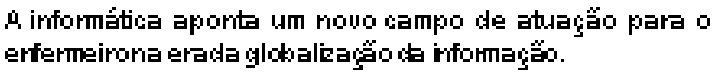 & 0,610 \\
\hline 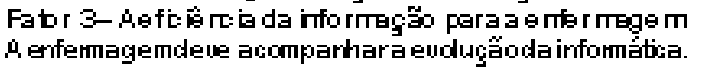 & 0,837 \\
\hline 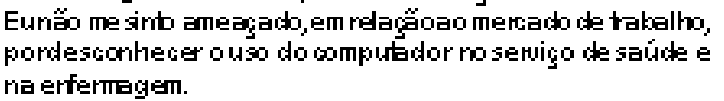 & 0,859 \\
\hline o computachornấo me assusta nem me embaraça. & 0,576 \\
\hline
\end{tabular}

Observa-se ainda que quatro fatores foram destacados da amostra dos estudantes, ao passo que três fatores emergiram da amostra dos enfermeiros. Os fatores 3 e 4 da amostra de estudantes sugerem uma relativa consistência interna, apresentando carga fatorial entre suas variáveis superiores a 0,600. A análise dos itens da carga fatorial do fator 2 da amostra dos estudantes sugere que ela é menor do que a da amostra dos enfermeiros, embora apresente alguns itens idênticos.

Para uma melhor compreensão da análise fatorial, vários testes foram realizados aplicando-se o método de rotação varimax. Procurou-se, assim, encontrar o agrupamento de variáveis que representassem um claro entendimento, isso porque a maior dificuldade no uso desse método é a interpretação dos resultados de acordo com a realidade da amostra. Assim sendo, pode-se verificar que a extração de quatro fatores para a amostra dos estudantes e de três para a amostra dos enfermeiros reflete a melhor interpretação. Além disso, em ambas as amostras, foram apresentados fatores que, no geral, totalizaram mais de $50 \%$ de variância.

0 resultado deste estudo sugere que estudantes e enfermeiros têm até certo ponto, uma atitude positiva em relação ao computador. Essas averiguações são coerentes com outros estudos $^{(3,6-7)}$. Embora a amostra dos estudantes demonstre que eles têm mais experiência com o computador, não houve uma diferença significativa na média de atitudes entre os dois grupos estudados. Percebe-se ainda que há uma atitude de moderação em relação ao uso do computador, ou seja, não é negativa, mas também não se pode afirmar que existe domínio de conhecimento e familiaridade com a operacionalização das informações computadorizadas.

Está claramente delineado, nos resultados apresentados, que os estudantes geralmente têm mais experiência com 0 computador do que os enfermeiros. Essa fato se explica pela introdução de conhecimentos de informática na formação desses futuros profissionais e também pelo surgimento de uma nova cultura de informação automatizada que leva as pessoas a buscarem esse conhecimento para competirem no mercado de trabalho que exige um novo perfil profissional. A geração de enfermeiros, atualmente inseridos no ambiente de trabalho, não teve as mesmas oportunidades de conhecer o computador e utilizá-lo no dia-a-dia. Na realidade, esses profissionais estão aprendendo a manusear esse equipamento em sua unidade de trabalho, alterando sua rotina para se adaptar aos novos instrumentos de trabalho.

É importante destacar ainda que da amostra envolvendo os estudantes emergiu um quarto fator que não foi identificado na amostra dos enfermeiros. Este caracterizou-se por aglomerar variáveis que refletem a necessidade de educação em informática. Isso implica uma atitude positiva dos estudantes, pois começam a perceber que o computador é uma ferramenta importante de trabalho, que precisa ser conhecido e explorado para melhor assistir o paciente e gerenciar o serviço. Entretanto, para o profissional, é preciso investir em tecnologia e treinamento de pessoal para se criar uma cultura positiva em relação ao uso do computador como instrumento que irá auxiliar o pessoal de Enfermagem a desenvolver suas atividades com 
eficiência e eficácia, aumentando o tempo de resolutividade e performance gerencial do serviço e do cuidado de Enfermagem ao paciente.

A Figura 1 sintetiza o conjunto das atitudes dos estudantes e enfermeiros apresentando a média geral de atitudes. Isso deixa claro que não há diferença de atitudes entre as duas amostras. Esse entendimento sugere a necessidade de se investir na educação desses profissionais em relação ao computador, preparando-os para uma mudança irreversível nos métodos de trabalho, em virtude do avanço da tecnologia da informação.

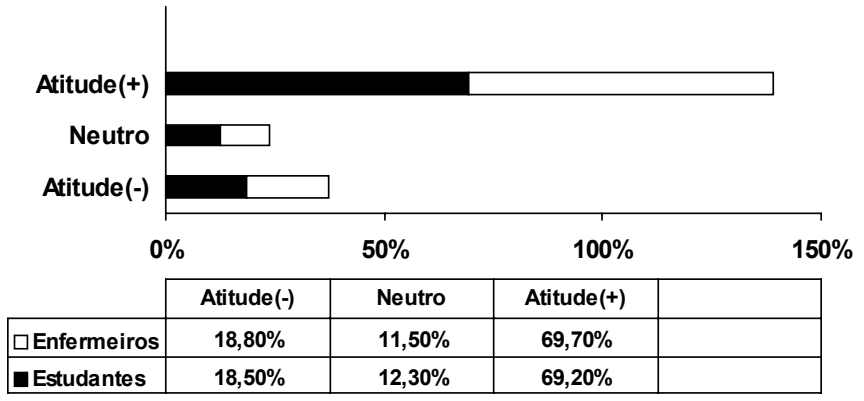

Figura 1 - Síntese das atitudes de estudantes ( $n=157)$ e enfermeiros $(n=80)$ em percentual médio

Esta nova realidade está sendo percebida pelos estudantes que, embora demonstrem um pouco mais de experiência com a informática, têm procurado cursar disciplinas que abordem a temática, através da oferta curricular, de caráter optativo. Naturalmente, compreende-se que o enfermeiro do terceiro milênio deverá ser 0 profissional que trabalhará a informação para gerenciar os cuidados de enfermagem.

\section{REFERÊNCIAS BIBLIOGRÁFICAS}

1. Jayasuriya R, Caputi P. Computer attitude and computer anxiety in nursing validation of na instrument using an Australian sample. Computers Nurs 1996 Nov/Dec; 14(6):304-5.

2. Gagne RM, Briggs L. Principles of instructional design. New York: Holt, Rinehart and Winston; 1979.

3. Schwirian PM, Malone JÁ, Stone VJ, Nunley B, Francisco T. Computers in nursing practice: a comparison of attitudes of nurses and nursing students. Computers Nurs 1989; 7:168-77.

4. Scarpa R, Smeltzer SC, Jasion B. Attitudes of nurses toward computerization: a replication. Computers Nurs $1992 \mathrm{Mar} / \mathrm{Apr}$; 10(2):72-80.

5. Stockton AH, Verhey MP. A psychometric examination of the Stronge-Brodt nurses' attitudes towards computers questionnaire. Computers Nurs 1995; 13:109-13.

6. Stronge JH, Brodt A. Assessment of nurses' attitudes towards computerization. Computers Nurs 1985; 4:154-8.

\section{CONCLUSÃO}

Para o enfermeiro na Paraíba, o computador ainda é um instrumento estranho à sua prática de trabalho. Não há um envolvimento contínuo com a informática, uma vez que são poucos os hospitais informatizados, embora se possa ver em muitos serviços de saúde um microcomputador no balcão de atendimento ao público. Neste estudo, verificou-se que tanto os enfermeiros quanto os estudantes apresentaram uma atitude positiva, conforme foi constatado através da análise fatorial e confirmado no gráfico de percentual médio de respostas. Estatisticamente, não houve diferença significativa nos escores das médias, embora se constate que a amostra de estudantes demonstre mais experiência e familiaridade com o computador do que a de enfermeiros.

A aplicação da análise fatorial, que é uma ferramenta estatística poderosa, foi usada neste estudo para conduzir a extração de componentes principais de análise pelo método de rotação varimax, sobre os itens do questionário. Para a amostra de estudantes, foram extraídos quatro fatores: fator 1 - barreiras em relação ao computador, fator 2 - o computador como ferramenta de trabalho; fator 3 - a eficiência da informação para enfermagem; fator 4 - necessidade de educação em informática. Para a amostra de enfermeiros, foram extraídos três fatores: fator 1 - barreiras em relação ao computador, fator 2 - o computador como ferramenta de trabalho; fator 3 - a eficiência da informação para enfermagem.

7. Bongartz C. Computer-oriented patient care: a comparison of nurses' attitudes perceptions. Computers Nurs 1988; 6:204-10.

8. Burkes M. Identifying and relating nurses' attitudes toward computer use. Computers Nurs 1991; 9:190-210.

9. Santos SR, Valdevino J Neto, Costa SPR. Qualidade de vida no trabalho do enfermeiro. Rev UNIPÊ 1998; 2(2):96 - 106.

10. Mcbride SH, Nagle LM. Attitudes toward computers - a test of construct validity. Computers Nurs 1996 May/June; 14(3):164-70.

11. Luis MAV, Moala FA, Évora YFM, Scochi CGS, Rodrigues RAP. Avaliação de uma uma disciplina de informática por graduandos de enfermagem. Rev Latino-am Enfermagem 1995 julho; 3(2):69-82.

12. Ministério da Saúde (BR). Conselho Nacional de Saúde. Comissão Nacional de Ética em Pesquisa - CONEP. Resolução no 196/96. Dispõe sobre pesquisa envolvendo seres humanos. Brasília (DF): Ministério da Saúde; 1996.

13. Norusis MJ. SPSS/PC+. Professional statistics. Chicago: SPSS Inc.; 1992.

14. Santos SR, Vilela MASD. Perfil do estudante de Enfermagem. Rev UNIPÊ 1997; 1(2):88-97. 